\title{
SOME QUANTITATIVE RESULTS RELATED TO ROTH'S THEOREM: CORRIGENDA
}

\author{
E. BOMBIERI AND A. J. VAN DER POORTEN
}

(Received 2 February 1989)

Theorem 1 of the paper [1] is stated in an unnecessarily weak form. What is actually proved is much stronger.

TheOREM 1. Let $\alpha_{1}, \ldots, \alpha_{n}$ be elements of a number field $K$ of degree $r$ over the field $k$ with each $\alpha_{i}$ of exact degree $r$ over $k$. Suppose $n \geq c_{0} \log r$ (where $c_{0}$ is a sufficiently large constant) and set $\eta: 0<\eta \leq 1 / 2 n !$. Let $\beta_{i} \in k$ be approximations to $\alpha_{i}, i=1, \ldots, n$, such that we have the gap condition

$$
\frac{1}{\eta} \log \left(4 h\left(\alpha_{i+1}\right)\right)+\log \left(4 h\left(\beta_{i+1}\right)\right) \geq \frac{4 r n}{\eta}\left(\frac{1}{\eta} \log \left(4 h\left(\alpha_{i}\right)\right)+\log \left(4 h\left(\beta_{i}\right)\right)\right) .
$$

Then

$$
\left|\alpha_{i}-\beta_{i}\right|_{v} \geq\left(\left(4 h\left(\alpha_{i}\right)\right)^{1 / \eta} 4 h\left(\beta_{i}\right)\right)^{-2-3 \sqrt{\log r} / \sqrt{n}}
$$

for at least one $i, 1 \leq i \leq n$.

The following misprints should be corrected: page 236 , line 3

$$
\text { for: } \tau=1 \quad \text { read: } \tau=n
$$

page 237, definition of $T(t)$

$$
\text { for: } t \leq x_{1} \leq 1 \quad \text { read: } t ; 0 \leq x_{i} \leq 1
$$

page 238 , middle

$$
\text { for: } U(z v(z)) \quad \text { read: } U(v(z))
$$

page 238, Lemma 5

$$
\text { for: } \sin \text { read: }
$$

(C) 1990 Australian Mathematical Society $0263-6115 / 90 \$ A 2.00+0.00$ 
[2] Some quantitative results related to Roth's theorem: Corrigenda

page 239, proof of Lemma 6

$$
\text { for: }>n / 2-n z \quad \text { read: }>n / 2+n z \text {. }
$$

\section{References}

[1] E. Bombieri and A. J. van der Poorten, 'Some quantitative results related to Roth's Theorem', J. Austral. Math. Soc. (Ser. A) 45 (1988), 233-248.

Institute for Advanced Study

Macquarie University

Princeton, New Jersey 08540

NSW 2109

U.S.A.

Australia 